GEOPHYSICAL RESEARCH LETTERS, VOL. 20, NO. 23, PAGES 2735-2738, DECEMBER 14, 1993

\title{
ION MEASUREMENTS DURING PIONEER VENUS REENTRY: IMPLICATIONS FOR SOLAR CYCLE VARIATION OF ION COMPOSITION AND DYNAMICS
}

\author{
J. M. Grebowsky1, R. E. Hartle' ${ }^{1}$ J. Kar ${ }^{1,2}$, P. A. Cloutier ${ }^{3}$, H. A. Taylor Jr. ${ }^{4}$ and L. H. Brace ${ }^{5}$
}

Abstract. During the final, low solar activity phase of the Pioneer Venus mission, the Orbiter Ion Mass Spectrometer measurements found all ion species, in the midnight-dusk sector, reduced in concentration relative to that observed at solar maximum. Molecular ion species comprised a greater part of the total ion concentration as $\mathrm{O}^{+}$and $\mathrm{H}^{+}$had the greatest depletions. The nightside ionospheric states were strikingly similar to the isolated solar maximum "disappearing" ionospheres. Both are very dynamic states characterized by a rapidly drifting plasma and $30-100 \mathrm{eV}$ superthermal $\mathrm{O}^{+}$ions.

\section{Introduction}

The first in situ measurements of the ion composition of the Venus ionosphere (e.g., Taylor et al., 1979, 1980), were obtained from the Pioneer Venus Orbiter Ion Mass Spectrometer (OIMS) during conditions of high solar activity. Pioneer Venus' periapsis altitude was deep in the ionosphere during the initial three years of the mission beginning December 1978 during a peak in the solar cycle, when the solar F10.7 index averaged near 200 (units: $10^{-22} \mathrm{~W} \mathrm{~m}-2 \mathrm{~Hz}^{-1}$ ). Again, for a period of approximately 50 days prior to the final destructive spacecraft entry into the atmosphere of Venus (October 8, 1992), the orbit traversed altitudes below $200 \mathrm{~km}$. During these final ionosphere traversals solar activity was decreasing towards a solar cycle minimum with an average F10.7 at Venus' longitude of approximately 120.

Radio occultation measurements provided glimpses of the ionospheric structure during the previous solar minimum (1984-1986) when the spacecraft was orbiting completely outside of the ionosphere (e.g., Knudsen et al., 1987; Kliore et al., 1991). They found that the solar minimum nightside ionosphere was severely depleted in comparison to solar maximum conditions. It was demonstrated (Knudsen et al. 1987) that the decrease was the result of a reduction in the net amount of ionization transported from the dayside into the night due to movement of the ionopause boundary closer to the planet. The OIMS preentry measurements provided the opportunity to look more closely at the solar activity changes.

\footnotetext{
1NASA/Goddard Space Flight Center

2 Now at National Physical Laboratory, India

3Rice University

${ }^{4}$ Taylor Enterprises, Nova Scotia

5University of Michigan

Copyright 1993 by the American Geophysical Union.
}

Paper number 93GL02239

0094-8534/93/93GL-02239\$03.00

\section{Solar Maximum \& Minimum Nighttime Ion Composition}

The lowest altitude passes of Pioneer Venus during the preentry period were located in the midnight to dawn local time (LT) sectors. Periapsis dropped below $200 \mathrm{~km}$ at about midnight local time. Periapsis subsequently drifted in local time to $0430 \mathrm{LT}$ when communication with the spacecraft ceased. For the current study, OIMS data from orbits with peripsis local times between 0000 and 0400 LT were selected. These were compared with ODMS measurements from the first three years of the mission in the same local time range. This restricted the data to the $\mathrm{LT}$ region of the hydrogen bulge, where neutral $\mathrm{H}$ as well as $\mathrm{H}^{+}$concentrations in the ionosphere maximize (Taylor et al., 1980; Brinton et al., 1980). This LT window also avoided mixing deep nightside distributions with the large horizontal ion density gradients that occur near the terminator. The altitude range was restricted to $140-400 \mathrm{~km}$.

Figures 1 and 2 depicit the average ion concentrations obtained from the 1979-1981 solar maximum OIMS data and from the 1992 preentry measurements. The plots include data from both inbound and outbound legs and hence presumably reflect altitude trends unaliased by horizontal latitudinal variations (PVO's periapsis latitude was $\sim 16^{\circ} \mathrm{N}$ for the solar maximum orbits and $\sim 10^{\circ} \mathrm{S}$ for the solar minimum period). The most prominent solar cycle change seen is the decrease in $\mathrm{O}^{+}$by more than an order of magnitude from solar maximum to minimum. The light ion $\mathrm{H}^{+}$, which in the $\mathrm{H}$ bulge region is produced via charge exchange (e.g., Brinton et al, 1980) between $\mathrm{H}$ and $\mathrm{O}^{+}$transported from the dayside, is the next most affected ion species. Its concentration drops on average by a factor of 4 . For both solar cycle periods the major ions $\mathrm{O}^{+}$and $\mathrm{H}^{+}$are comparable in magnitude above $200 \mathrm{~km}$. (It is characteristic at Venus that an Earth-like protonosphere with negligible concentrations of heavier ions never prevails.) The small displacements of the $\mathrm{O}^{+}$averages above and below those of $\mathrm{H}^{+}$are not statistically significant - the standard deviations of the data scatter, for all ion species, are factors of 3-4 about the means. The absence of similar large solar cycle changes in the other ion species indicates: 1. the importance of ionization sources that are not related to the transport of ionization from daylight into the night, and 2. significant differences in ion chemistry from species to species. Details of the processes can be found in the comprehensive discussion of nightside ionosphere chemistry of Fox (1992). The drop in total ion concentration is consistent with earlier radio occultation measurements for similar solar activity levels but is not yet as low as the densities detected by occultation experiments at still lower activity (e.g., Kliore et al., 1991 and references therein).

Under solar minimum conditions the importance of a source of ionization in addition to transport from the dayside has been demonstrated by Knudsen et al. (1987) and more recently, using the preentry OIMS measurements, by Kar et al. (1993). 
PIONEER-VENUS OIMS
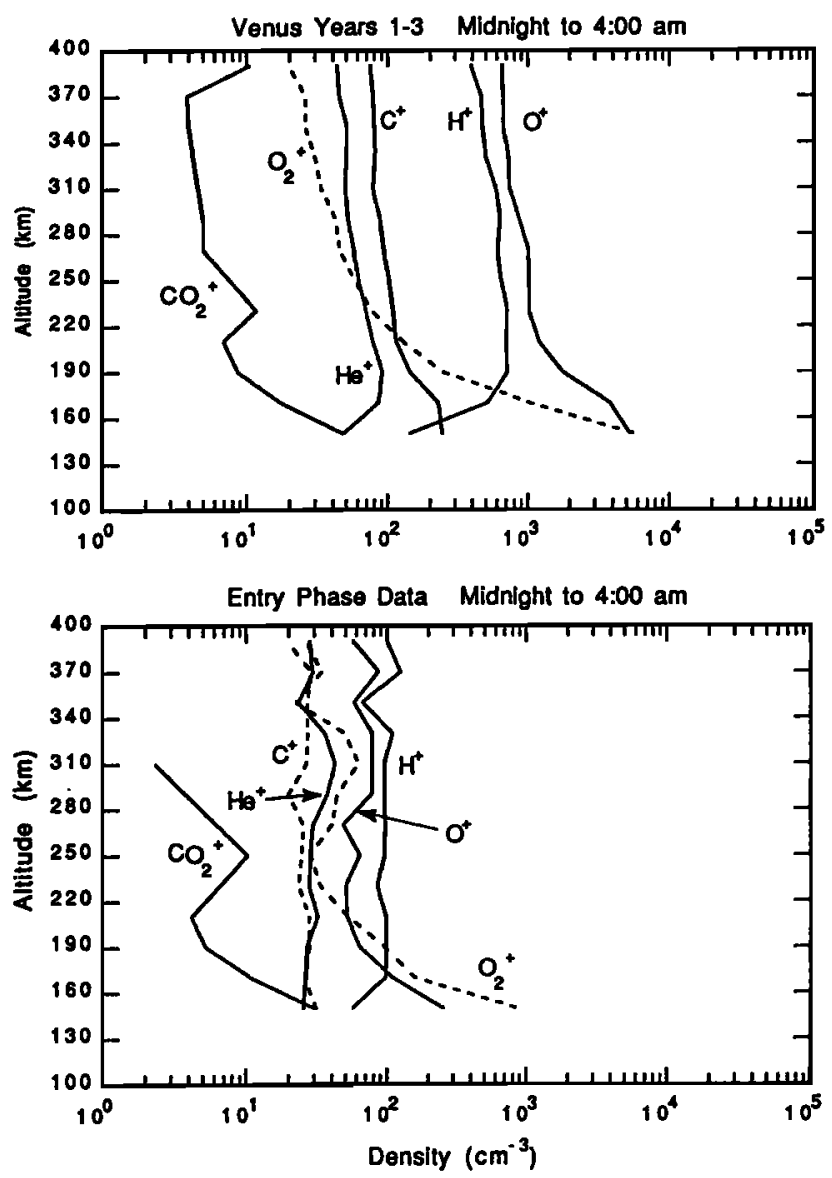

Fig. 1. Average ion concentrations (from the $12 \mathrm{~s}$ averaged Unified Abstract Data System) for 6 ion species in $20 \mathrm{~km}$ altitude bins between 140 and $400 \mathrm{~km}$. Top: solar maximum measurements. Bottom: the low solar activity reentry data.

These studies presented evidence that the average nightside ionospheric layer of $\mathrm{O}_{2}{ }^{+}$(as well as $\mathrm{N}_{2}{ }^{+} / \mathrm{CO}^{+}$) observed at solar minimum could be attributed predominantly to energetic electron impact ionization. This is in contrast to the typical solar maximum state where the transported $\mathrm{O}^{+}$has been shown to be the dominant source for the nightside $\mathrm{O}_{2}{ }^{+}$layer (e.g., Taylor et al., 1980; Spenner et al., 1981). However, as Fox and Taylor (1990) deduced for $\mathrm{N}_{2}{ }^{+}$, impact ionization still can play a significant role in the ion composition at times even during solar maximum. The atomic ion species $\mathrm{N}^{+}, \mathrm{C}^{+}$and $\mathrm{He}^{+}$, like $\mathrm{O}^{+}$, in the nightside ionosphere also have sources attributable to the transport of ionization from daylight. Of course, their eventual evolution on the nightside is not coupled to the distribution of neutral $\mathrm{H}$, as is $\mathrm{O}^{+}$which encounters a significant ionization sink in traversing the hydrogen bulge. Hence their changes through the solar cycle, as observed, do not parallel that of $\mathrm{O}^{+}$. The most general change in composition from solar maximum to minimum is an increase in the relative concentration of the molecular ions due to the reduction of the dominant atomic ion concentrations.

The minor species profiles $\left(\mathrm{O}^{++}, \mathrm{O}_{18^{+}}, \mathrm{D}^{+}\right.$and the high altitude $\mathrm{CO}_{2}^{+}$) should be taken as upper limits to the true ambient average concentrations. The detection sensitivity of the OIMS to ambient ions drifting at spacecraft speed ranges from $\sim 4$ to $\sim 8 \mathrm{~cm}^{-3}$ depending on spacecraft angle of attack and species mass. When the means of the measured concentrations approach these background thresholds they begin to lose their statistical significance. The $\mathrm{D}^{+}$measurements were the most sensitive of all masses with a threshold of $\sim 1 \mathrm{~cm}^{-3}$.

\section{Disappearing Ionospheres}

Although the average ion concentration profiles in Figures 1 and 2 show significant trends, they wash out the range of complex ionospheric states (e.g., Taylor et al., 1980) that have been encountered. For example, in the early mission solar maximum observations, smooth ion density variations along the PVO orbit would be interrupted by sharp bite outs in the ion densities associated with the development of features such as ionospheric troughs or "holes". (e.g., Brace et al., 1982). Even more spectacular are the cases, first noted by Taylor et al. $(1979,1980)$ in which the entire nightside ionosphere segment along the orbit is severely depleted. These "disappearing" ionospheres (Cravens et al., 1982) are imbedded with ordered and enhanced magnetic fields compared to the typical ionosphere state. Hence, within the solar maximum statistics in Figures 1 and 2 exists a subset of orbits with reduced plasma densities and properties distinctly different from the nominal filled-up ionospheric states that dominate the distributions. The OIMS observations in conjunction with electron density

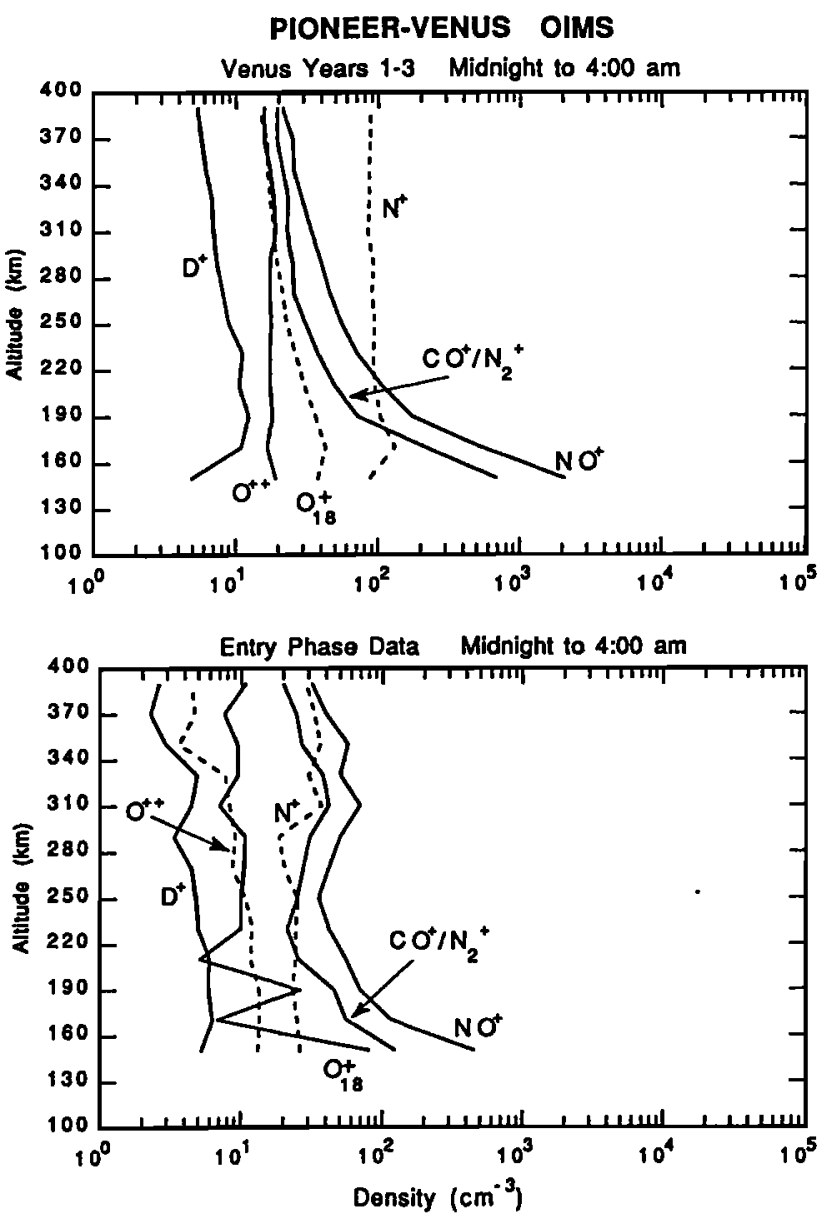

Fig. 2. Average ion concentrations of the remaining 6 ion species. 


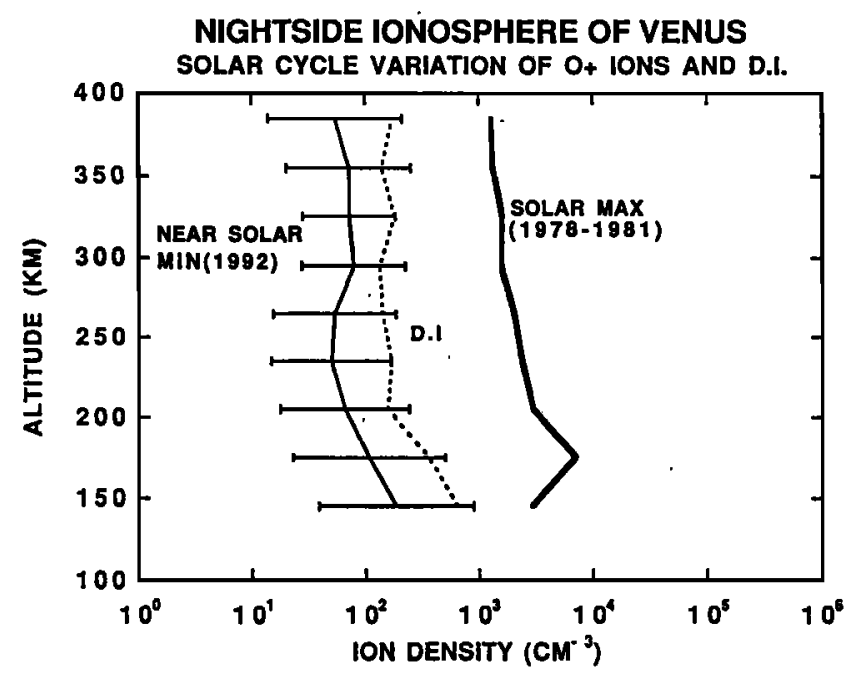

Fig. 3. Average $\mathrm{O}^{+}$concentrations in $30 \mathrm{~km}$ bins in the range 0000-0430 LT for all Pioneer Venus solar maximum, minimum, and "disappearing" ionospheres. The standard deviations, similar in magnitude for all groups, is depicted for the solar minimum data.

measurements from the Orbiter Electron Temperature Probe (OETP) offer evidence that the ionospheric states near solar minimum were characteristically similar to the solar maximum "disappearing" ionospheres and that a distinct change in the overall nightside ionosphere dynamics takes place with decreasing solar activity.

Figure 3 compares the average altitude distributions of the OIMS measurements of $\mathrm{O}^{+}$for the near solar minimum observations on the dawnside of midnight to the average solar maximum measurements in the same local time range. In addition it displays the average of the subset of the solar maximum data corresponding to disappearing ionosphere cases. The disappearing ionospheres were defined as those states in which the ion density above the main ionosphere peak was severely depleted with concentrations generally below $1000 \mathrm{~cm}^{-3}$. A total of 26 disappearing ionosphere cases were included. Most of these solar maximum orbits are listed in Luhmann (1992). It is seen that for both the solar minimum and solar maximum cases similar reductions occur in the $\mathrm{O}^{+}$ density. Both reductions presumably are caused by a reduction in the transport of ionization from the dayside when the solar wind pressure increases relative to the dayside ionosphere pressure. Disappearing ionospheres occur under solar maximum conditions when the solar wind pressure increases above nominal values. During low solar activity, the reduction of dayside ion production with a nominal solar wind dynamic pressure produces a similar effect.

One of the prominent features of the disappearing ionospheres at solar maximum is the presence of an ordered B field which often has a horizontal inclination, in contrast to the signature of fields directed parallel to the sun-earth line that have been used to characterize the isolated holes (e.g., Luhmann, 1992). Unfortunately 3-dimensional B field measurements were not available in the preentry period. However other characteristic features, referred to but never expanded upon by Cravens et al. (1982), are also present: high drift speeds and superthermal $\mathrm{O}^{+}$ions. These characteristics observed in the response of the OMMS will further demonstrate the identical behavior in the near solar minimum observations.

An indication of the presence of large scale drifts, through their impact on the OIMS response, is dramatically demonstrated in a few extreme ion profiles obtained within solar maximum disappearing ionospheres and the solar minimum nightside ionosphere. The measurements from inbound legs of two such orbits selected because of the remarkable similarity are shown in Figure 4. The plots show the concentrations of the 3 major ion species observed on inbound orbital legs for a near solar minimum orbit on top and a depleted ionosphere solar maximum observation on the bottom. The electron density observed by the OETP in the topside ionosphere is consistently higher than the total of the OIMS derived ion densities. This apparent discrepancy is attributed to high ion flow velocities which causes some ion concentrations not to be measured accurately. The ion concentrations were derived by assuming that the ions enter the spectrometer with the spacecraft velocity. If the ion drift velocity is large, and not aligned with the spacecraft velocity vector, the resultant angle of attack will differ from that assumed to convert the measured current to concentration. This affects the heavier ions more than the light ions due to the higher thermal speeds of the latter and their resultant larger acceptance angle. The ion concentrations deduced in the
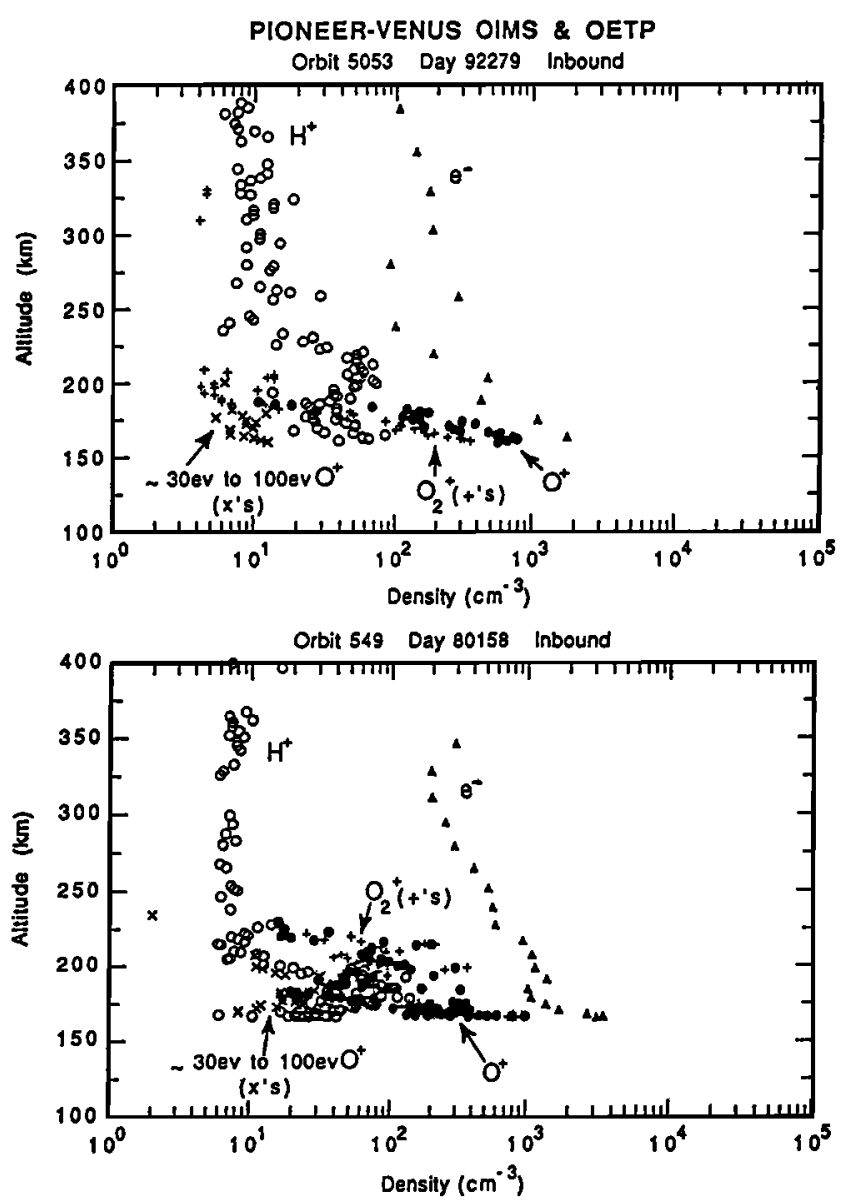

Fig. 4. Inbound leg OIMS high resolution measurements and $12 \mathrm{~s}$ averaged OETP electron density data for a "disappearing" ionosphere from solar maximum, on the bottom, and a similarly distributed end of mission profile on top. 
standard data processing will be low if the ion flow direction into the OIMS is at a significantly greater incident angle than ions entering along the spacecraft velocity line.

The measurements in Figure 4 show no $\mathrm{O}^{+}$detection for altitudes above $\sim 200 \mathrm{~km}$. The most plausible explanation of the data is the $\mathrm{O}^{+}$ions are drifting. An ambient flow velocity directed orthogonal to the OIMS orifice, or from the wake of the satellite, with a speed comparable to the spacecraft speed of - $10 \mathrm{~km} / \mathrm{s}$ could produce the observed response. No explanation other than the presence of ambient drifts can currently be offered for this behavior.

A rough estimate of changing flow conditions with solar activity can be obtained from the relationship between the electron density and OIMS derived total ion concentration. The ratio of the electron density to the total ion concentration for the 0000-0400 LT near solar minimum measurements between 200 and $400 \mathrm{~km}$ has a geometrical mean of 3 . For the solar maximum data the mean is only 1.6. The solar maximum ratio, which increases with increasing altitude, is consistent with the horizontal $\mathrm{O}^{+}$speeds of $1-4 \mathrm{~km} / \mathrm{s}$ measured by the Orbital Retarding Potential Analyzer (e.g., Knudsen et al. 1980) with similar speeds inferred from OIMS measurements (Taylor et al., 1980). The near solar minimum, as well as the solar maximum disappearing ionospheres, require higher drift speeds to account for the OIMS-OETP derived density differences.

Another property of disappearing ionospheres cited by Cravens et al. (1982) was the existence of superthermal ions. The OIMS as described by Grebowsky et al. (1993) also has the capability of measuring the presence of superthermal $\mathrm{O}^{+}$ ions in the energy range of $\sim 30$ to $100 \mathrm{eV}$ The solar minimum observations reveal traces of these ions at all altitudes in the topside of the ionosphere. Similar behavior is also detected at solar maximum for disappearing ionosphere profiles but is absent in the full-up ionospheres. The source of these ions has yet to be determined.

\section{Conclusion}

The low solar activity, nightside Venus ionosphere has many similarities with solar maximum "disappearing" ionospheres. Both have low plasma densities, drifting plasmas and 30-100 $\mathrm{eV}$ superthermal $\mathrm{O}^{+}$ions.

Acknowledgments. Data aid was supplied by Joel Selekof (STX Systems). One of the authors, J. K., was an NRCNASA research associate when this work was performed.

\section{References}

Brace, L. H., R. F. Theis, H. G. Mayr, S. A. Curtis. and J. G. Luhmann, Holes in the nightside ionosphere of Venus, J. Geophys. Res., 87, 199-211, 1982.

Brinton, H. C., H. A. Taylor, Jr., H. B. Niemann, H. G. Mayr, A. F. Nagy, T. E. Cravens, and D. F. Strobel, Venus nighttime hydrogen bulge, Geophys. Res. Lett., 1, 865-868, 1980.

Cravens, T. E., L. H. Brace, H. A. Taylor, Jr., C. T. Russell, W. C. Knudsen, K. L. Miller, A. Barnes, J. D. Mihalov, F. L. Scarf, S. J. Quenon and A. F. Nagy, Disappearing Ionospheres on the nightside of Venus, Icarus, $\underline{51}, 271$ 282, 1982.
Fox, J. L., Chemistry of the nightside ionosphere of Venus, Planet. Space Sci.., 40, 1663-1681, 1992.

Fox, J. L. and Taylor, H. A. Jr., A signature of electron precipitation in the nightside ionosphere, Geophys. Res. Lett., 17 1625-1628, 1990.

Grebowsky, J. M., W. T. Kasprzak, R. E. Hartle, K. K. Mahajan, and T. C. G. Wagner, Superthermal ions detected in Venus' dayside ionosheath, ionopause, and magnetic barrier regions, J. Geophys. Res., 98, 9055-9064, 1993.

Kar, J., R. E. Hartle, J. M. Grebowsky, and P. A. Cloutier, Evidence of the dominance of electron impact ionization in producing the nightside ionosphere peak from PVO/OIMS measurements at solar minimum, Abstract, 1993 Spring AGU meeting, EOS. 74, 186, 1993.

Kliore, A. J., J. G. Luhmann and M. H. G. Zhang, The effect of the solar cycle on the maintenance of the nightside ionosphere of Venus, J. Geophys. Res, 96, 11065-11071, 1991.

Knudsen, W. C., K. Spenner, K. L., K. L. Miller and V. Novak, Transport of $\mathrm{O}^{+}$ions across the Venus terminator and implications, $\mathrm{J}$ Geophys. Res., 85, 7803-7810, 1980.

Knudsen, W. C., A. J. Kliore, and R. C. Whitten, Solar Cycle Changes in the Ionization Source of the Nightside Venus Ionosphere, I. Geophys. Res., 92, 13391-13398, 1987.

Luhmann, J. G., Pervasive large scale magnetic fields in the Venus nightside ionosphere and their implications, J.Geophys. Res., 97, 6103-6121, 1992.

Spenner, K., W.C. Knudsen, R. C. Whitten, P. F. Michelson, K. L. Miller and V. Novak, On the maintenance of the Venus nightside ionosphere: Electron precipitation and plasma transport, J. Geophys. Res., 86, 9170-9178,1981.

Taylor, H. A. Jr., H. C. Brinton, S. J. Bauer, R. E. Hartle, P. A. Cloutier and R. E. Daniell, Global observations of the composition and dynamics of the ionosphere of Venus: Implications for the solar wind interaction, $\mathbf{L}$ Geophys.Res., 85, 7765-7777, 1980.

Taylor, H. A. Jr., H. C. Brinton, S. J. Bauer, R. E. Hartle, P. A. Cloutier and R. E. Daniell, and Donahue, T. M., Ionosphere of Venus: First observations of day-night variation of the ion composition, Science 205, 96-99, 1979.

J. M. Grebowsky, R. E. Hartle, Code 914, NASA, Goddard Space Flight Center, Greenbelt, MD 20771

J. Kar, Radio Science Div., National Physical Laboratory, Hillside Road, New Delhi-110012, India.

P. A. Cloutier, Dept. of Physics \& Astronomy, Rice University, P.O. Box 1892, Houston, TX 77251

H. A. Taylor, Jr., Taylor Enterprises, R.R. \#1, Box 2495, Beaver River, Nova Scotia, B5A 4A5

L. H. Brace, U. Michigan, 2455 Hayward, Ann Arbor, MI 48109

(Received July 6, 1993;

accepted July 29, 1993) 